\title{
Alterations of brain metrics in fetuses of women with polycystic ovary syndrome : a retrospective study based on fetal magnetic resonance imaging
}

Zhongkun $\mathrm{Bao}^{\dagger}$, Qing Zhang ${ }^{\dagger}$, Manman Pan, Xi Xi, Yuanlin Wang, Fangfang Zhang, Fangfang Wang, Yu Zou ${ }^{*}$ and Fan Qu*

\begin{abstract}
Background: Maternal polycystic ovary syndrome (PCOS) has potential detrimental effects on the neurodevelopment of offspring. This study aimed to evaluate the brain metrics in fetuses of women with PCOS based on fetal magnetic resonance imaging (MRI).

Methods: This retrospective study included 60 pregnant women with PCOS (PCOS group) and 120 pregnant nonPCOS women (control group). Fetal MRI was performed followed an ultrasound and for numerous clinical indications including known or suspected fetal pathology, history of fetal abnormality in previous pregnancy or in a family member. Fetal brain biometry and apparent diffusion coefficient (ADC) value were analysed.

Results: After adjusting for potential confounders, fetuses in the PCOS group showed the following characteristics compared to fetuses in the control group: (1) smaller cerebral fronto-occipital diameter (FOD), vermian height (VH) and anteroposterior diameter of the pons (APDP) (evident before 32 weeks; $P=0.042, P=0.002$ and $P=0.016$, respectively); (2) larger left and right biparietal index (evident before 32 weeks; $P=0.048$ and $P=0.025$, respectively); (3) smaller left lateral ventricle (LV) (evident after 32 weeks; $P=0.005$ ); (4) larger anteroposterior diameter of the vermis (APDV) and hippocampal infolding angle (HIA) (evident after 32 weeks; $P=0.003$ and $P<0.001$, respectively); (5) higher ADC value in frontal white matter (FWM) and in basal ganglia (BG) (evident before and after 32 weeks; all $P<0.05$ ).
\end{abstract}

Conclusions: There exist a different pattern of brain metrics in PCOS offspring in utero.

Keywords: Apparent diffusion coefficient, Brain biometry, Fetus, Magnetic resonance imaging, Polycystic ovary syndrome

\footnotetext{
* Correspondence: zouyuzju@zju.edu.cn; syqufan@zju.edu.cn

${ }^{\dagger}$ Zhongkun Bao and Qing Zhang contributed equally to this study. Women's Hospital, School of Medicine, Zhejiang University, 1 Xueshi Road,

Zhejiang 310006 Hangzhou, China
}

(c) The Author(s). 2021 Open Access This article is licensed under a Creative Commons Attribution 4.0 International License, which permits use, sharing, adaptation, distribution and reproduction in any medium or format, as long as you give appropriate credit to the original author(s) and the source, provide a link to the Creative Commons licence, and indicate if changes were made. The images or other third party material in this article are included in the article's Creative Commons licence, unless indicated otherwise in a credit line to the material. If material is not included in the article's Creative Commons licence and your intended use is not permitted by statutory regulation or exceeds the permitted use, you will need to obtain permission directly from the copyright holder. To view a copy of this licence, visit http://creativecommons.org/licenses/by/4.0/. The Creative Commons Public Domain Dedication waiver (http://creativecommons.org/publicdomain/zero/1.0/) applies to the data made available in this article, unless otherwise stated in a credit line to the data. 


\section{Background}

Polycystic ovary syndrome (PCOS) occurs in 5-12\% of women at reproductive age [1]. With the characteristics of hyperandrogenism, irregular menstruation and ovulatory dysfunction, PCOS accounts for $80 \%$ of the cause of anovulatory infertility [2]. As transgenerational influences of maternal PCOS are potentially imparted to the offspring, an increasing attention is paid to the health of PCOS offspring [3]. The physical development, neurodevelopment, metabolic and reproductive profiles, endocrine status, cardiovascular feature of PCOS offspring have been presented [4]. The genetic component and the intrauterine environment of maternal PCOS, may have adverse impact on programming and developing of PCOS offspring [5]. However, it is not clear whether the impacts of maternal PCOS on offspring exist from the early development in fetal period, and few has investigated fetal characteristics of PCOS in utero.

Fetal magnetic resonance imaging (MRI) is a useful medical imaging modality for assessing fetal pathology, especially for fetal neurologic evaluation. Relying primarily on T2-weighted sequence, MRI examination done during pregnancy could assess the in vivo fetal brain maturation from 18th week of gestation to term [6]. Based on fetal and placental MRI, we have previously found that there exist alterations of fetal physical growth and development, together with decreased placental thickness in women with PCOS [7]. Considering the potential influence of maternal PCOS on neurodevelopmental health of offspring, the study hypothesized that the alteration in neurodevelopment of offspring may origin in fetal life in utero and the offspring of women with PCOS may show abnormal changes in brain biometry and cerebral microstructure. To verify the hypothesis, this study measured brain biometry and apparent diffusion coefficient (ADC) value in fetuses of women with PCOS by fetal MRI.

\section{Methods}

\section{Study design and participants}

This retrospective study included singleton pregnancies of women who attended fetal MRI and delivered with live birth infants in our hospital. The Ethics Committee approved this study with written informed consent obtained before all fetal MRI procedures. The hospital's radiology database were queried for fetal MR imaging of totally 4132 pregnant women between 2013 and 2018 . The clinical indications for fetal MRI include known or suspected fetal pathology (posterior fossa anomaly, corpus callosal anomaly, diaphragmatic hernia, microcephaly, isolated ventriculomegaly, cleft lip/palate, etc.) followed an ultrasound examination, and history of fetal abnormalities in family members or in a previous pregnancy. We excluded 2122 pregnant women because of presence of pre-existing maternal diseases (including diabetes, chronic hypertension, thyroid disease, etc.), twin or multiple pregnancies, history of maternofetal infection, abnormal fetal karyotype, any associated fetal body or brain abnormality, delivery with stillbirth, and cases in which the fetal brain was not scanned or with poor MRI image quality.

PCOS was defined according to the Rotterdam 2003 consensus criteria [8] with two of three features: anovulation, polycystic ovarian morphology on ultrasound, and hyperandrogenism. For those who had isolated PCOS feature and the information provided in database could not help to determine a PCOS diagnosis, they were telephoned and asked to participate to a survey about their gynaecological history. They would be included into PCOS group if they told they had history of previous PCOS diagnosis and provided details diagnosing PCOS in the telephone visit. As a control group, data from pregnant healthy women without PCOS were used. These women had a history of regular menstrual cycles and no clinical and/or biochemical signs of hyperandrogenism. For each women in PCOS group, two controls with a similar gestational age (GA) at the time of MRI examination were randomly selected during the same period. Finally, we identified fetal MRI examinations from 60 pregnant women with diagnosed PCOS and 120 control pregnant women. Figure 1 showed the flow chart of the sampling frame and study population.

\section{Magnetic resonance imaging}

The fetus MRI was operated using a 1.5-Telsa (T) scanner (Signa HDxt, General Electric Company, CT, USA) with a phased- array abdominal coil. The single-shot fast spin echo (SSFSE: short repetition time/echo time (TR/ TE), 3100/90 ms; bandwidth, $32 \mathrm{kHz}$; field-of-view (FOV), $30 \times 32 \mathrm{~cm}$; matrix, $256 \times 192$; slice thickness, 5 $\mathrm{mm}$; gap, $0 \mathrm{~mm}$; number of excitations (NEX), 1) sequence weighted images in the sagittal, axial and frontal planes were used to the maternal uterus. The single-shot fast spin echo (SSFSE: TR/TE, 3100/90 ms; bandwidth, $32 \mathrm{kHz}$; FOV, $30 \times 32 \mathrm{~cm}$; matrix, $256 \times 192$; slice thickness, 4-5 mm; gap, $0 \mathrm{~mm}$; NEX, 1) and fast imaging employing steady-state acquisition (FIESTA: TR/TE, 3.6/ $1.7 \mathrm{~ms}$; bandwidth, $80 \mathrm{~Hz}$; FOV, $32 \times 32 \mathrm{~cm}$; matrix, $256 \times 224$; slice thickness, $5 \mathrm{~mm}$; gap, $0 \mathrm{~mm}$; flip angle, $\left.55^{\circ}\right)$ sequences on all three planes were used to the fetal brain. Diffusion-weighted imaging (DWI) of the fetal brain was performed in the axial plane with single-shot echo-planar sequence (TR/TE, 3200/88 ms; FOV, $36 \mathrm{~cm} \times 36 \mathrm{~cm}$; slice thickness, $4 \mathrm{~mm}$; b-value, $600 \mathrm{~s} /$ $\mathrm{mm} 2$ ). All sequences were performed with the specific absorption ratio (SAR) values lower than $2.0 \mathrm{~W} / \mathrm{kg}$. 


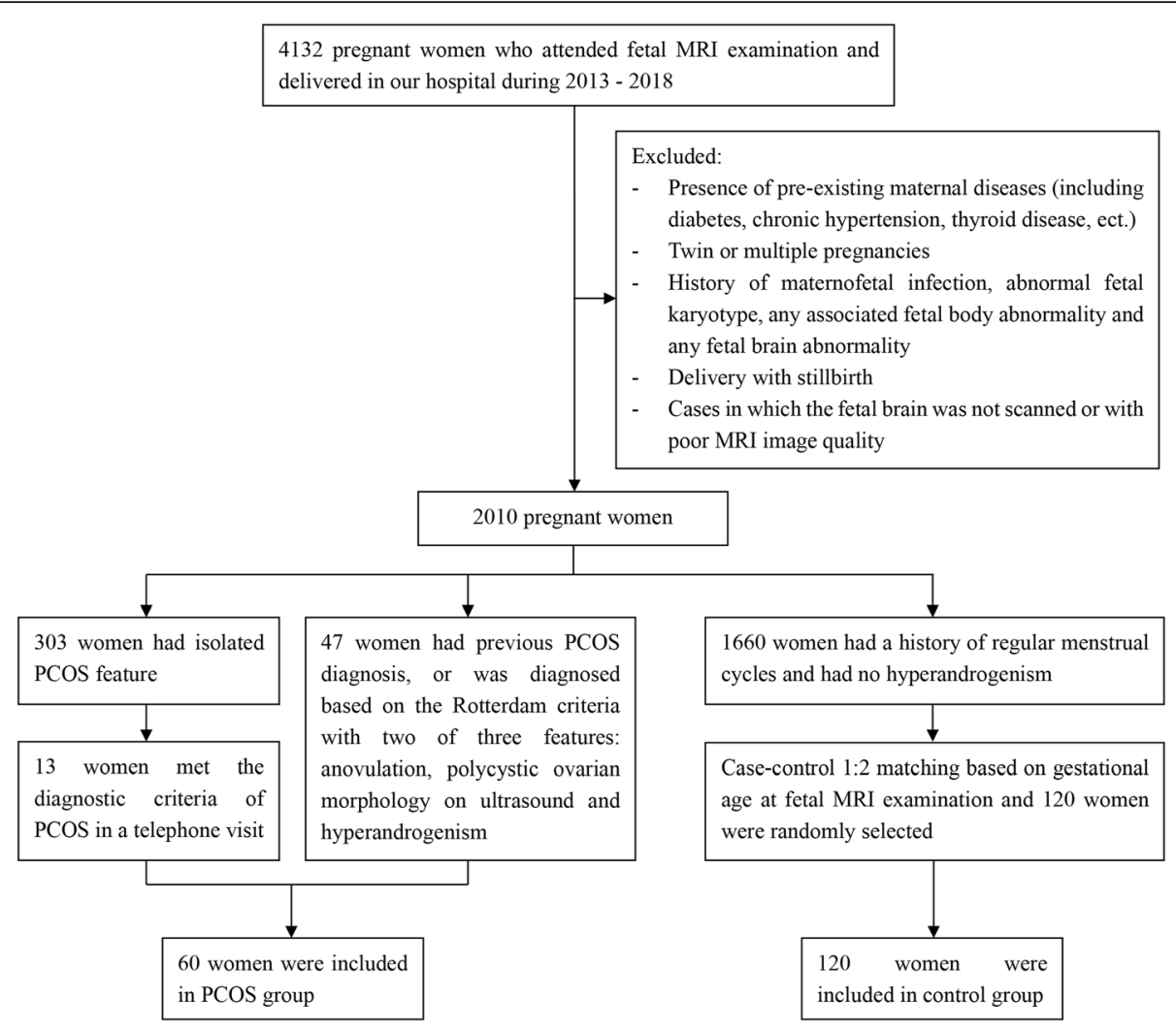

Fig. 1 Flow chart of the sampling frame and study population. MRI, magnetic resonance imaging; PCOS, polycystic ovary syndrome

\section{Data collection}

Measurements of the brain biometry and ADC value were made independently by two radiologists experienced in fetal MRI and blinded to participants' information. The following fetal brain biometric parameters were measured: bone and cerebral fronto-occipital diameters (FODs), bone and cerebral biparietal diameters (BPDs), transverse cerebellar diameter (TCD), anteroposterior diameter of the vermis (APDV), vermian height (VH), hippocampal infolding angle (HIA), length of the corpus callosum (LCC), anteroposterior diameter of the pons (APDP) and lateral ventricles (LV). ADC values in frontal white matter (FWM) and in basal ganglia (BG) were obtained from DWI section, and ADC maps were reconstructed using the AW workstation and "Functool" software version 9.4.05a. The methodology used to measure fetal brain biometric parameters and ADC value was followed with previous studies [9-12] and detailed in Fig. 2. Maternal and infant demographic and clinical characteristics were also collected from the hospital's electronic medical record system.

\section{Study size}

We included data of pregnancy women with PCOS and non-PCOS controls with a similar GA at MRI examination between 2013 and 2018. Since the lack of published data on the best fetal brain MRI biometric parameters to use as primary end-point, power calculation was not feasible and the study size was determined according to the maximum number of eligible participants in consideration of the experimental design of the study.

\section{Statistical analysis}

A Kolmogorov-Smirnov test was performed for the normality of continuous variable data. The Student's $t$ test was utilized for normally distributed quantitative data and the Mann-Whitney $U$ test for non-parametric data. Categorical variables were analysed by Chi-squared test or Fisher exact tests. To analyse inter-group differences in fetal brain metrics, multivariate linear regression analysis was used with PCOS as independent and each fetal brain biometric parameter or ADC value as dependent variable. $P$-values resulting from univariate analyses were subsequently adjusted for potential confounders, including maternal age, body mass index (BMI), mode of conception, GA at MRI examination, and the presence of gestational diabetes mellitus (GDM), pre-eclampsia and preterm birth. The quadratic nonlinear analyses were used to indicate the correlation between GA and fetal brain biometric MRI parameters. For all analyses, twosided $P$-values of $<0.05$ was considered statistically significant. The SPSS version 20 (IBM, Armonk, NY, USA) 

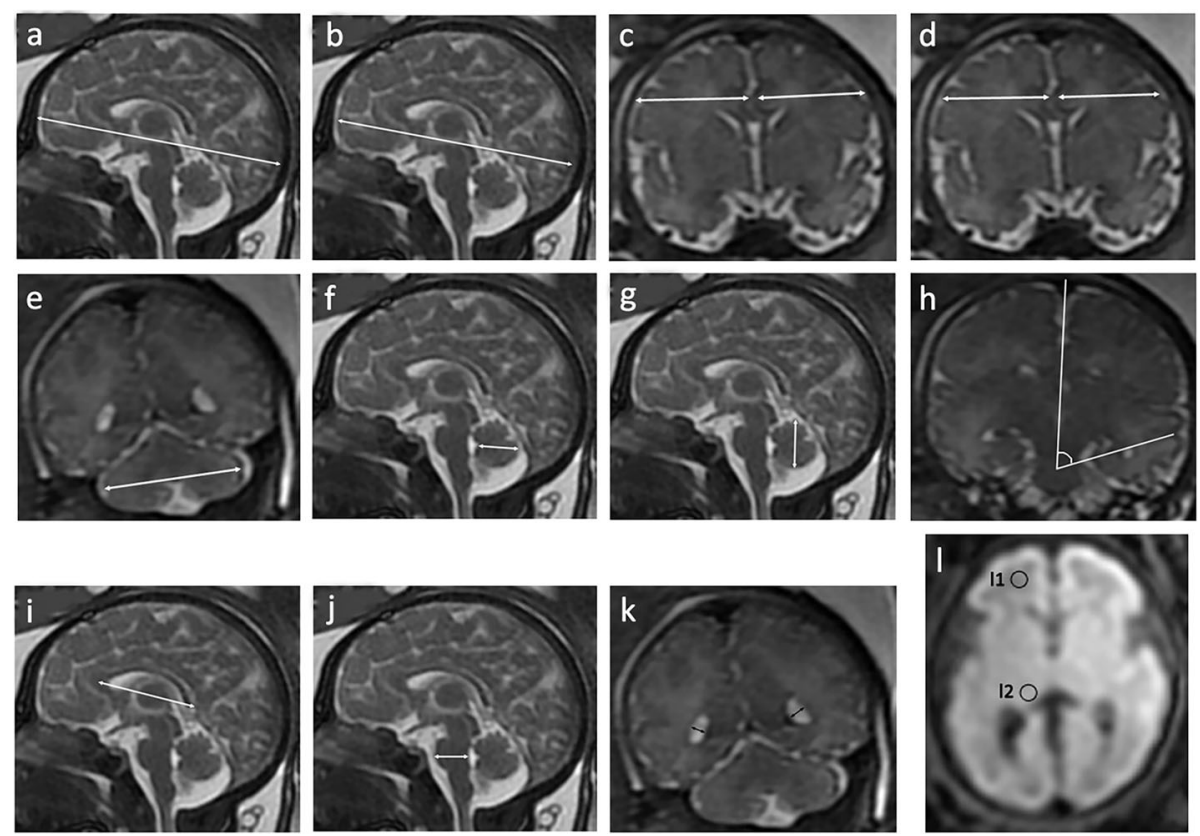

Fig. 2 Magnetic resonance imaging slice showing measurement of: (a) bone fronto-occipital diameter; (b) cerebral fronto-occipital diameter; (c) left and right bone biparietal diameters; (d) left and right cerebral biparietal diameters; (e) transverse cerebellar diameter; (f) anteroposterior diameter of the vermis; (g) vermian height; (h) hippocampal infolding angle; (i) length of the corpus callosum; (j) anteroposterior diameter of the pons; (k) left and right lateral ventricles; and (I) apparent diffusion coefficient measures in frontal white matter (I1) and in basal ganglia (I2)

was used for all statistical analyses and statistical significance was set at a level of $P<0.05$.

\section{Results}

Baseline and clinical characteristics of study population As showed in Fig. 3, the median GA at fetal MRI examination was 32 weeks for both groups (interquartile range: 30,35 in PCOS group and 30, 34 in control group), without a significant difference in distribution between the two groups $(P=0.707)$. Therefore, in the subsequent comparative analysis, sub-groups were stratified into GA $\leq 32$ weeks and GA > 32 weeks. There was no significant difference in maternal demographic data between PCOS and control groups except the mode of conception $(P<0.001)$. The rate of preterm birth $(<37$ weeks) in PCOS group were significantly higher than that of the controls $(P=0.002)$. The details of baseline characteristics and clinical data were shown in Table 1.

\section{Fetal brain biometric MRI parameters and the correlation with GA}

As shown in Table 2, fetal brain biometric MRI parameters in PCOS group when GA $\leq 32$ weeks and $>32$ weeks were compared with the control group. And Table 3 showed the results of univariate analysis and subsequently multivariate linear regression analysis to adjust for potential confounders. When $G A \leq 32$ weeks, fetal $\mathrm{VH}$ and APDP in PCOS group was significantly smaller than control group ( $P=0.018,0.027$, respectively). After adjustment for potential confounding variables, cerebral FOD, VH and APDP of fetuses in PCOS group were significantly smaller than control group $(P=0.042,0.002$, 0.016 , respectively), while left and right biparietal index in PCOS group were significantly larger than control group $(P=0.048,0.025$, respectively). When $\mathrm{GA}>32$ weeks, left bone BPD and left cerebral BPD of fetuses in PCOS group were significantly smaller than control group $(P=0.034,0.036$, respectively), however, after adjusting potential confounders these differences were not statistically significant. Fetuses in PCOS group had significantly smaller left LV $(P=0.001)$ and significantly larger APDV and HIA $(P=0.006,0.000$, respectively) than control group, and these were still significant after adjusting potential confounders $(P=0.005,0.003,0.000$, respectively).

For the GA range of 20-39 weeks, a nonlinear quadratic model was the best fit for the change values of all the biometric parameters except left and right LV. Quadratic regression analyses suggested a significant association between GA and these parameters in both groups (all $P<0.01$ ) (Fig. 4).

\section{ADC value and the correlation with GA}

There were 53 in PCOS group and 116 in control group with DWI available among the overall fetal MRI examinations. After adjusting for potential confounders, ADC 


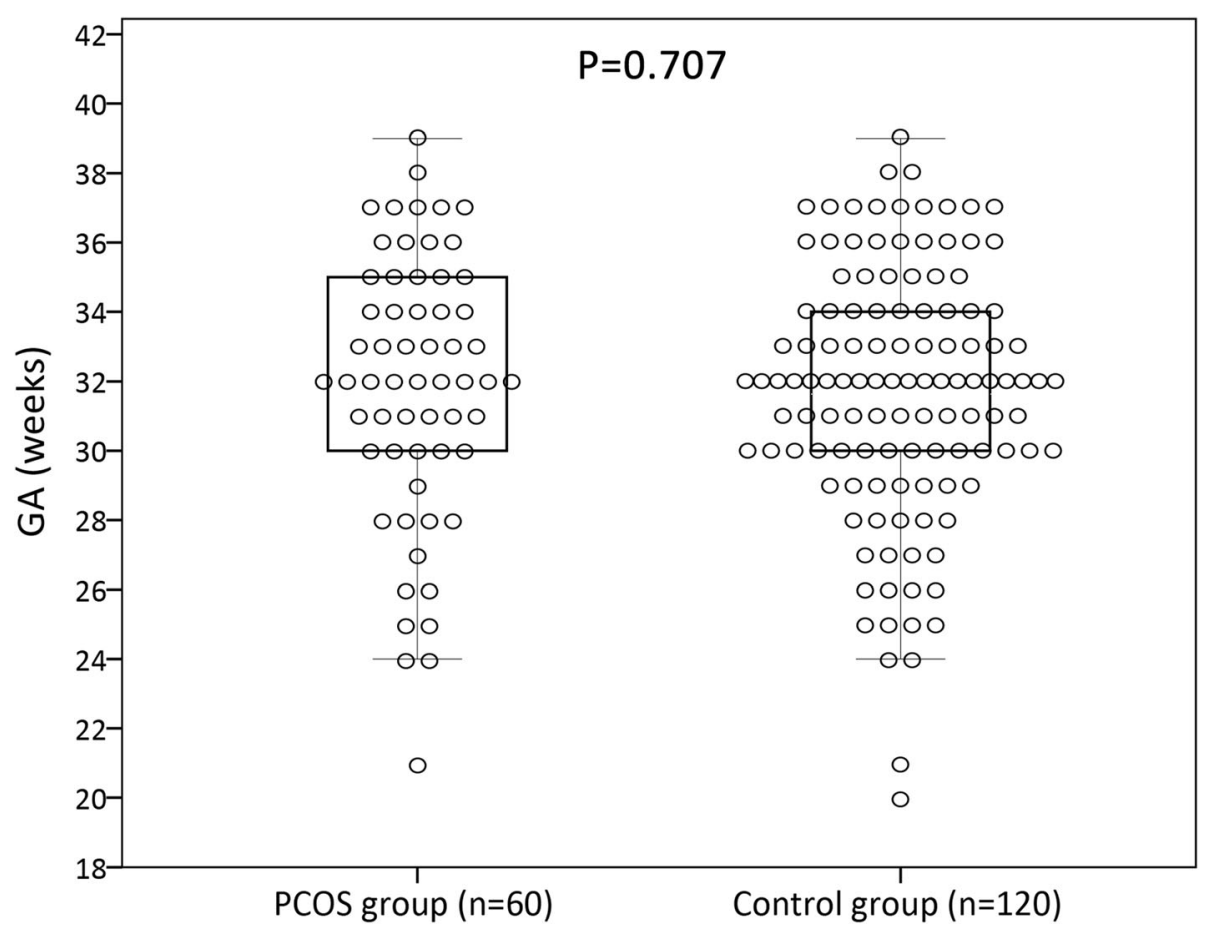

Fig. 3 Distributions of gestational age (GA) at fetal MRI examination in polycystic ovary syndrome (PCOS) and control groups. Comparison was determined by the Mann-Whitney $U$ test

value in FWM and in BG in PCOS group was significantly higher than the control group both when $\mathrm{GA} \leq 32$ weeks $(P=0.024,0.032$, respectively $)$ and when $\mathrm{GA}>32$ weeks ( $P=0.032,0.031$, respectively) (Fig. 5a-b). Quadratic regression analyses suggested a significant association between GA and ADC value of FWM in PCOS group $(P=0.010)$, and also between GA and ADC value of BG in the control group $(P<0.001)$ (Fig. $5 \mathrm{c}-\mathrm{d})$.

\section{Discussion}

The current study assessed the pattern of fetal brain growth in utero in offspring of women with PCOS by measuring brain MRI biometry and ADC value. Fetuses of women with PCOS appeared to show a smaller cerebral size and a larger peri-cerebral space, which was reflected by MRI measurements of smaller cerebral FOD and higher biparietal index than fetuses of women without PCOS. All these inter-group differences have been adjusted for confounding factors including baseline characteristics and pregnancy complications. Moreover, fetuses of women with PCOS have alteration in morphometry of cerebral structures, indicated by smaller VH, APDP and left LV, larger APDV and HIA, and elevated $A D C$ values in FWM and in BG.

Fetal brain biometric MRI parameters has become an approach to observe fetal growth [10]. Brain metrics at term-equivalent age displayed shorter bifrontal and biparietal diameters and TCD in preterm infants in contrast to full term infants [13, 14]. In fetuses of fetal growth restriction (FGR), the total brain, cerebral and cerebellar volumes were smaller compared to control fetuses [15]. Fetal BPD after 24 weeks' GA could be detected as a predictor of small-for-gestational age (SGA)[16]. Although he prevalence of SGA and FGR in our study was not different between groups, the small cerebral size and increased peri-cerebral space in fetuses of women with PCOS suggest a delayed brain growth and might be signs of FGR or SGA, or more prone to develop preterm birth, which support those studies reporting that new-borns of women with PCOS were more frequently to be delivered with SGA [17]. In accordance with the present result, a previous study has found that fetuses of women with PCOS exhibited smaller BPD at 32 weeks of GA and shorter body length at birth, indicating maternal PCOS has a potential restrictive effect on fetal growth and development [18].

The cerebellum plays an important part in motor tasks and numerous higher functions such as learning, memory, attention, cognition, and behaviour [19]. We found an abnormal cerebellar morphometry in fetuses of women with PCOS, as indicated by smaller $\mathrm{VH}$ and larger APDV of cerebellum. Although we failed to get the growth pattern of cerebellum in fetuses of women with PCOS, the opposite trend of alterations in $\mathrm{VH}$ and APDV of cerebellum may be associated with the change in cerebellar functions, as the different growth rates of 
Table 1 Baseline and clinical characteristics

\begin{tabular}{|c|c|c|c|}
\hline Items & PCOS group $(n=60)$ & Control group $(n=120)$ & $\begin{array}{l}\text { PCOS vs. Control } \\
P \text { value }\end{array}$ \\
\hline \multicolumn{4}{|l|}{ Mothers } \\
\hline Age (years) & $30.6 \pm 4.0$ & $30.3 \pm 4.0$ & 0.666 \\
\hline \multicolumn{4}{|l|}{ Pre-pregnancy BMI (kg/m²) } \\
\hline $\mathrm{BMI}<25$ & $53(88.3)$ & $112(93.3)$ & 0.264 \\
\hline $\mathrm{BMI} \geq 25$ & $7(11.7)$ & $8(6.7)$ & \\
\hline \multicolumn{4}{|l|}{ GA at MRI examination (weeks) } \\
\hline$\leq 32$ weeks & $33(55.0)$ & $73(60.8)$ & 0.453 \\
\hline$>32$ weeks & $27(45.0)$ & $62(39.2)$ & \\
\hline \multicolumn{4}{|l|}{ Current employment status [n(\%)] } \\
\hline Full-time employment & $49(81.7)$ & $102(85.0)$ & 0.562 \\
\hline Part-time employment & $5(8.3)$ & $9(7.5)$ & \\
\hline Registered unemployed & $6(10.0)$ & $7(5.8)$ & \\
\hline Others & $0(0.0)$ & $2(1.7)$ & \\
\hline \multicolumn{4}{|l|}{ Educational level [n(\%)] } \\
\hline Less than college education & $16(26.7)$ & $28(23.3)$ & 0.710 \\
\hline College education & $38(63.3)$ & $83(69.2)$ & \\
\hline Higher than college education & $6(10.0)$ & $9(7.5)$ & \\
\hline Gravidity (times) & $2(1,2)$ & $2(1,3)$ & 0.390 \\
\hline \multicolumn{4}{|l|}{ Parity [n(\%)] } \\
\hline Nulliparity & $40(66.7)$ & $74(61.7)$ & 0.512 \\
\hline Multiparity & $20(33.3)$ & $46(38.3)$ & \\
\hline \multicolumn{4}{|l|}{ Mode of conception [n (\%)] } \\
\hline Nature conception & $47(78.3)$ & $116(96.7)$ & 0.000 \\
\hline Pharmacological ovulation induction & $4(6.7)$ & $0(0.0)$ & \\
\hline In-vitro fertilization & $9(15.0)$ & $4(3.3)$ & \\
\hline GDM [n (\%)] & $14(23.3)$ & $15(12.5)$ & 0.062 \\
\hline Pre-eclampsia [n (\%)] & $5(8.3)$ & $3(2.5)$ & 0.073 \\
\hline $\mathrm{PIH}[\mathrm{n}(\%)]$ & $3(5.0)$ & $5(4.2)$ & 0.798 \\
\hline ICP [n (\%)] & $3(5.0)$ & $3(2.5)$ & 0.402 \\
\hline \multicolumn{4}{|l|}{ Mode of delivery [n (\%)] } \\
\hline Vaginal delivery & $28(46.7)$ & $69(57.5)$ & 0.169 \\
\hline Caesarean delivery & $32(53.3)$ & $51(42.5)$ & \\
\hline \multicolumn{4}{|l|}{ Offspring } \\
\hline Ultrasound biparietal diameter at MRI examination $(\mathrm{cm})$ & $8.09 \pm 0.86$ & $8.18 \pm 0.97$ & 0.566 \\
\hline Ultrasound femur length at MRI examination (cm) & $5.92 \pm 0.78$ & $5.99 \pm 0.83$ & 0.574 \\
\hline GA at birth (weeks) & $38.3 \pm 2.2$ & $38.8 \pm 1.5$ & 0.167 \\
\hline FGR & $1(1.7)$ & $3(2.5)$ & 0.721 \\
\hline Preterm birth (<34 weeks) & $3(5.0)$ & $1(0.8)$ & 0.074 \\
\hline Preterm birth (<37 weeks) & $10(16.7)$ & $4(3.3)$ & 0.002 \\
\hline \multicolumn{4}{|l|}{ Gender [n (\%)] } \\
\hline Male & $33(55.0)$ & $73(60.8)$ & 0.453 \\
\hline Female & $27(45.0)$ & $47(39.2)$ & \\
\hline Birth weight (g) & $3258.3 \pm 658.5$ & $3300.5 \pm 563.9$ & 0.656 \\
\hline
\end{tabular}


Table 1 Baseline and clinical characteristics (Continued)

\begin{tabular}{|c|c|c|c|}
\hline Items & PCOS group $(n=60)$ & Control group $(n=120)$ & $\begin{array}{l}\text { PCOS vs. Control } \\
P \text { value }\end{array}$ \\
\hline Birth height (cm) & $49.0 \pm 3.6$ & $49.7 \pm 2.5$ & 0.216 \\
\hline Birthweight <10th centile (SGA) & $2(3.3)$ & $5(4.2)$ & 0.785 \\
\hline Birthweight >90th centile (LGA) & $4(6.7)$ & $6(5.0)$ & 0.645 \\
\hline \multicolumn{4}{|l|}{1 min Apgar score [n (\%)] } \\
\hline$<7$ & $1(1.7)$ & $2(1.7)$ & 1.000 \\
\hline$\geq 7$ & $59(98.3)$ & $118(98.3)$ & \\
\hline \multicolumn{4}{|l|}{ 5min Apgar score [n (\%)] } \\
\hline$<7$ & $0(0.0)$ & $1(0.8)$ & 1.000 \\
\hline$\geq 7$ & $60(100.0)$ & $119(99.2)$ & \\
\hline NICU admission [n (\%)] & $28(46.7)$ & $41(34.2)$ & 0.104 \\
\hline
\end{tabular}

Note: Data was presented as mean \pm standard deviation (SD), median $\left(25^{\text {th }}, 75^{\text {th }}\right.$ quartiles) or number (percentage). Comparisons were determined by the Student's t-test and the chi-squared test. PCOS polycystic ovary syndrome, BMI body mass index, GA gestational age, MRI magnetic resonance imaging, GDM gestational diabetes mellitus, PIH pregnancy-induced hypertension, ICP intrahepatic cholestasis of pregnancy, FGR fetal growth restriction, SGA small-for-gestational age, $L G A$ large-for-gestational age, NICU neonatal intensive care unit

cerebellar subregions are related to their functional characteristics [20]. Furthermore, fetuses of women with PCOS presented increased HIA value after 32 gestational weeks, reflecting an abnormal hippocampal rotation process associated with hippocampal development or other cortical malformations [11]. However, it is unclear whether the altered cerebellar morphometry and HIA value, together with the decreased width of left LV in fetuses of women with PCOS found in the present study, is associated with the neurodevelopmental outcomes in postnatal life, which deserves further research.

ADC value decrease during fetal development, which is a quantitative approach to assess the morphologic brain maturity in utero. The increased ADC value in

Table 2 Comparisons of fetal brain biometric MRI parameters between PCOS group and control group

\begin{tabular}{|c|c|c|c|c|c|c|}
\hline \multirow[t]{2}{*}{ Parameters } & \multicolumn{2}{|l|}{$\begin{array}{l}\text { PCOS group } \\
(n=60)\end{array}$} & \multicolumn{2}{|l|}{$\begin{array}{l}\text { Control group } \\
(n=120)\end{array}$} & \multicolumn{2}{|c|}{$\begin{array}{l}\text { PCOS vs. Control } \\
P \text {-value }\end{array}$} \\
\hline & $\mathrm{GA} \leq 32$ weeks & GA >32 weeks & GA $\leq 32$ weeks & GA >32 weeks & $\mathrm{GA} \leq 32$ weeks & GA $>32$ weeks \\
\hline Bone FOD (mm) & $90.5 \pm 11.3$ & $102.1 \pm 6.0$ & $94.5 \pm 9.2$ & $104.4 \pm 6.6$ & 0.082 & 0.140 \\
\hline Cerebral FOD (mm) & $85.0 \pm 11.0$ & $97.8 \pm 6.2$ & $89.5 \pm 9.6$ & $100.2 \pm 7.0$ & 0.050 & 0.140 \\
\hline Fronto-occipital index & $0.06 \pm 0.02$ & $0.04 \pm 0.01$ & $0.05 \pm 0.02$ & $0.04 \pm 0.02$ & 0.104 & 0.550 \\
\hline Left bone BPD (mm) & $35.5 \pm 5.7$ & $39.7 \pm 3.0$ & $36.5 \pm 4.2$ & $41.5 \pm 3.5$ & 0.386 & 0.034 \\
\hline Left cerebral BPD (mm) & $31.9 \pm 5.0$ & $37.5 \pm 3.0$ & $33.2 \pm 4.3$ & $38.9 \pm 3.0$ & 0.176 & 0.036 \\
\hline Left biparietal index & $0.10 \pm 0.05$ & $0.06 \pm 0.02$ & $0.09 \pm 0.04$ & $0.06 \pm 0.03$ & 0.313 & 0.902 \\
\hline Right bone BPD (mm) & $35.0 \pm 5.1$ & $39.9 \pm 2.5$ & $36.1 \pm 4.4$ & $41.3 \pm 3.5$ & 0.258 & 0.070 \\
\hline Right cerebral BPD (mm) & $31.6 \pm 4.7$ & $37.8 \pm 2.7$ & $33.2 \pm 4.5$ & $38.6 \pm 3.2$ & 0.113 & 0.223 \\
\hline Right biparietal index & $0.10 \pm 0.04$ & $0.06 \pm 0.02$ & $0.08 \pm 0.04$ & $0.06 \pm 0.03$ & 0.096 & 0.143 \\
\hline TCD (mm) & $35.5 \pm 6.4$ & $45.9 \pm 4.6$ & $37.4 \pm 5.6$ & $46.7 \pm 3.9$ & 0.151 & 0.427 \\
\hline $\mathrm{VH}(\mathrm{mm})$ & $15.8 \pm 2.6$ & $19.1 \pm 2.3$ & $17.2 \pm 2.9$ & $20.1 \pm 2.4$ & 0.018 & 0.081 \\
\hline APDV (mm) & $13.0 \pm 2.2$ & $16.4 \pm 2.4$ & $12.6 \pm 2.2$ & $15.0 \pm 1.7$ & 0.395 & 0.006 \\
\hline LCC (mm) & $38.4 \pm 5.9$ & $41.3 \pm 4.5$ & $39.1 \pm 4.8$ & $42.2 \pm 3.7$ & 0.516 & 0.391 \\
\hline APDP (mm) & $10.5 \pm 1.9$ & $13.3 \pm 1.3$ & $11.3 \pm 1.6$ & $13.4 \pm 1.1$ & 0.027 & 0.630 \\
\hline Left LV (mm) & $8.2 \pm 3.0$ & $6.2 \pm 2.0$ & $7.7 \pm 2.4$ & $7.9 \pm 2.1$ & 0.387 & 0.001 \\
\hline Right LV (mm) & $7.7 \pm 2.5$ & $6.5 \pm 2.1$ & $7.3 \pm 2.4$ & $7.4 \pm 2.5$ & 0.472 & 0.112 \\
\hline $\mathrm{HIA}\left({ }^{\circ}\right)$ & $71.6 \pm 5.0$ & $75.2 \pm 4.5$ & $69.9 \pm 4.1$ & $71.6 \pm 3.4$ & 0.076 & 0.000 \\
\hline
\end{tabular}

Note: Data was presented as mean \pm standard deviation (SD). Comparisons were determined by Student's t-test. PCOS polycystic ovary syndrome, GA gestational age, $F O D$ fronto-occipital diameter, $B P D$ biparietal diameter, $T C D$ transverse cerebellar diameter, $V H$ vermian height, $A P D V$ anteroposterior diameter of the vermis, $L C C$ length of the corpus callosum, APDP anteroposterior diameter of the pons, $L V$ lateral ventricle, $H I A$ hippocampal infolding angle, $B M I$ body mass index, MRI magnetic resonance imaging, GDM gestational diabetes mellitus 


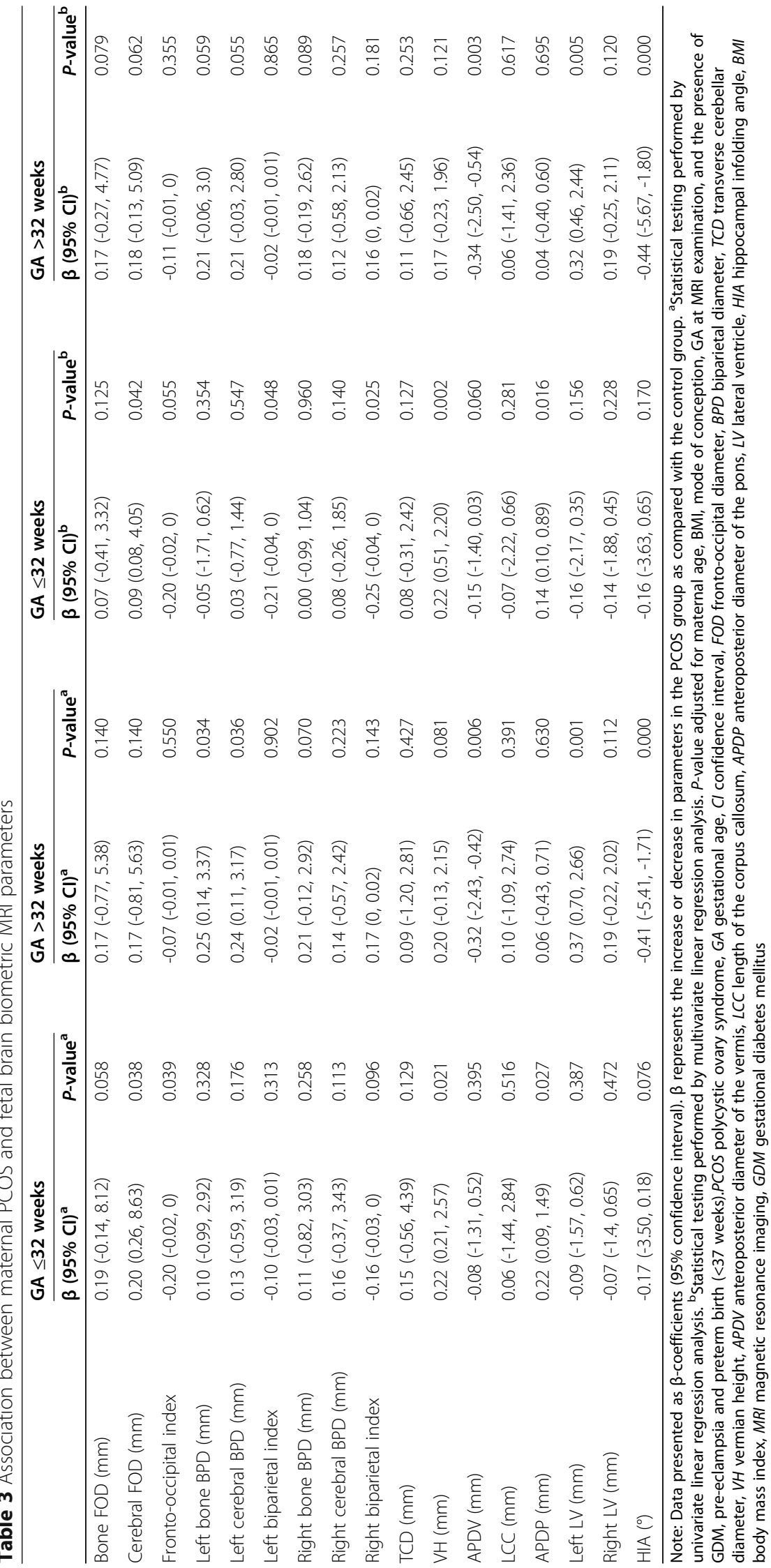




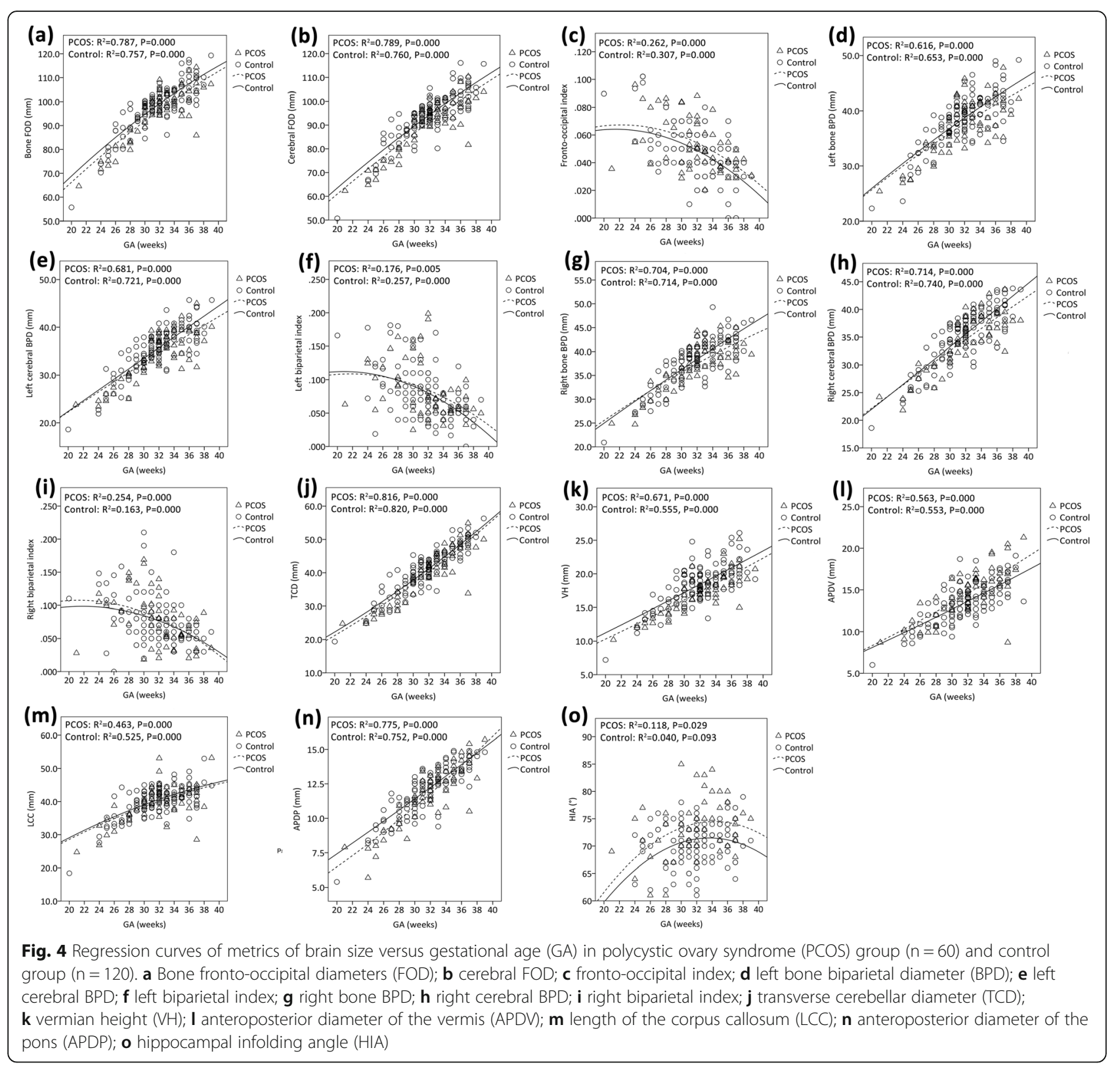

fetal brain is associated with aberrant signal intensity in white matter which may indicate brain histologic injuries such as the presence of vasogenic edema and astrogliosis [21]. The BG is anatomically near to the lateral ventricles and $\mathrm{ADC}$ value in $\mathrm{BG}$ is theoretically more prone to be influenced by a raised pressure in the ventricle[22]. However, this theory does not explain the decreased width of left LV in fetuses of women with PCOS found in the present study. Thus, we assumed that mechanisms other than intraventricular pressure cause elevation of the ADC in the BG.

The implications of different pattern of fetal brain metrics for later neurocognitive development are not yet clear. However, previous studies found that brain metrics of preterm new-borns could predict their developmental level of cognitive and motor function at 2 years of corrected age [14]. The alterations in morphometry of cerebellum and brain stem in SGA fetuses were correlated with neurocognitive and neurobehavior during the neonatal phase [23]. Thus, the different brain biometric MRI parameters in fetuses of women with PCOS compared to fetuses of non-PCOS women in this study, may provide the hint to the neurodevelopmental disorders that occur in postnatal life. As several population-based studies have reported, maternal PCOS may have broader detrimental impacts on neurodevelopment of the offspring, resulting in increased risk for developmental delay [24], pervasive 


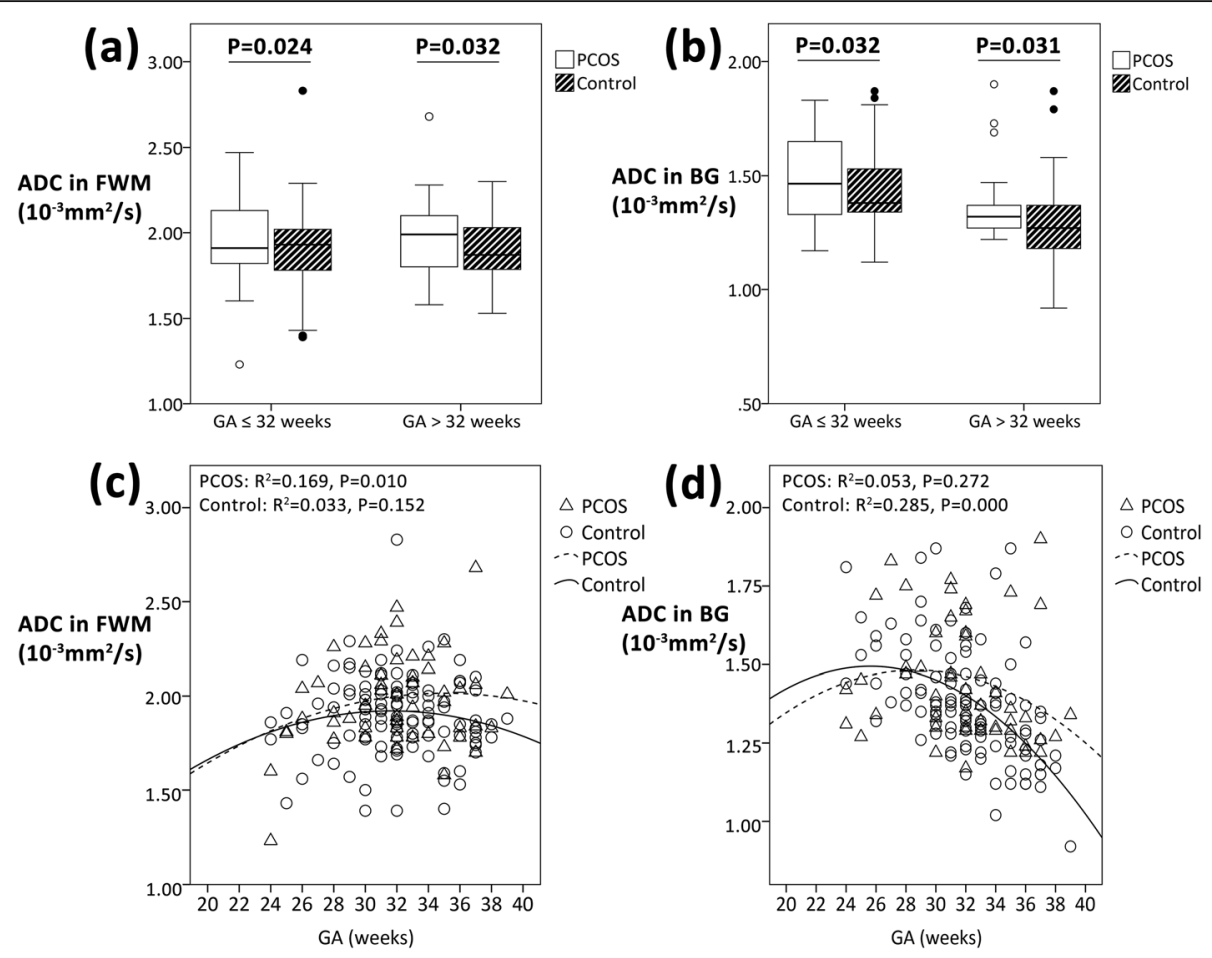

Fig. 5 Comparisons of ADC value in FWM (a) and in BG (b) between PCOS group and control group when GA $\leq 32$ weeks (PCOS: $n=31 ;$ control: $n=69$ ) and > 32 weeks (PCOS: $n=22$; control: $n=47$ ), P-value adjusted for maternal age, BMl, mode of conception, GA at MRI examination, and the presence of GDM, pre-eclampsia and preterm birth ( $<37$ weeks). And regression curves of ADC value in FWM (c) and in BG (d) versus GA in PCOS group $(n=53)$ and control group $(n=116)$. ADC, apparent diffusion coefficient; FWM, frontal white matter; BG, basal ganglia; PCOS, polycystic ovary syndrome; GA, gestational age; BMI, body mass index; MRI, magnetic resonance imaging; GDM, gestational diabetes mellitus

developmental disorders (PPDs)[25], autism spectrum disorders (ASD) and attention-deficit/hyperactivity disorder (ADHD)[26].

Although the present study provides the first biometric MRI evidence concerning alterations of in utero brain metrics of fetuses of PCOS women, the conclusion appears limited because of the small sample size and the retrospective study design. Lack of 3D reconstruction of fetal brain makes it failed to conduct super resolutionbased volumetric measurements. And the association of PCOS phenotype and the presence of fetal brain abnormalities needs further investigation. Limitations of the study also include the absence of unmeasurable images for some of the cases and the lack of long-term followup data for neurodevelopment outcomes. Nevertheless, the implication of our findings is ensured by the robust design, high-quality fetal MRI measurements and comprehensive data collection. In addition, as maternal prenatal characteristics and pregnancy complications were reported to be associated with human fetal brain development [27-30], in this study all results were adjusted for various potential confounders such as maternal age, prenatal BMI, mode of conception, and the presence of GDM, pre-eclampsia and preterm birth, making the cohorts comparable.

\section{Conclusions}

In conclusion, the present study found a different pattern of brain metrics in PCOS offspring in utero measured by fetal MRI.

\section{Abbreviations}

PCOS: Polycystic ovary syndrome; MRI: Magnetic resonance imaging; ADC: : Apparent diffusion coefficient; GA: Gestational age; DWI: Diffusion-weighted imaging; FOD: Fronto-occipital diameter; BPD: Biparietal diameter;

TCD: Transverse cerebellar diameter; APDV: Anteroposterior diameter of the vermis; VH: Vermian height; HIA: Hippocampal infolding angle; LCC: Length of the corpus callosum; APDP: Anteroposterior diameter of the pons; LV: Lateral ventricles; FWM: Frontal white matter; BG: Basal ganglia; BMI: Body mass index; GDM: Gestational diabetes mellitus; SD: Standard deviation; PIH: Pregnancy-induced hypertension; ICP: Intrahepatic cholestasis of pregnancy; NICU: Neonatal intensive care unit; FGR: Fetal growth restriction; SGA: Small-for-gestational age; LGA: Large-for-gestational age; PPD: Pervasive developmental disorder; ASD: Autism spectrum disorder; ADHD: Attentiondeficit/hyperactivity disorder

\section{Acknowledgements}

Not applicable.

\section{Authors' contributions}

$F Q$ and $Y Z$ designed the research, reviewed and edited the manuscript. ZKB and QZ wrote the manuscript and performed data analysis. MMP, XX, YLW and FFZ collected and analyzed the data. FFW explained the data. All authors read and approved the final manuscript. 


\section{Funding}

This study was supported by the National Natural Science Foundation of China under Grant 81874480 and 81873837; and Zhejiang Province Science Foundation for Distinguished Young Scholars under Grant LR16H040001.

\section{Availability of data and materials}

The datasets analysed during the current study are available from the corresponding author on reasonable request.

\section{Declarations}

\section{Ethics approval and consent to participate}

The Ethics Committee of Women's Hospital, School of Medicine, Zhejiang University, approved the study with an ethical approval number 2019-006. All procedures followed were in accordance with the ethical standards of the responsible committee on human experimentation (institutional and national) and with the Helsinki Declaration of 1975. Written informed consent was obtained before all fetal magnetic resonance imaging procedures.

\section{Consent for publication}

Not applicable.

\section{Competing interests}

The authors declared that they have no competing interests.

Received: 18 February 2021 Accepted: 20 July 2021

Published online: 14 August 2021

\section{References}

1. Bozdag G, Mumusoglu S, Zengin D, Karabulut E, Yildiz BO. The prevalence and phenotypic features of polycystic ovary syndrome: a systematic review and meta-analysis. Hum Reprod. 2016; 31:2841-55.

2. Balen AH, Morley LC, Misso M, Franks S, Legro RS, Wijeyaratne CN et al. The management of anovulatory infertility in women with polycystic ovary syndrome: an analysis of the evidence to support the development of global WHO guidance. Hum Reprod Update. 2016; 22:687-708.

3. Hart R. Generational Health Impact of PCOS on Women and their Children. Med Sci (Basel). 2019;7:1-8.

4. Vanky E, Engen Hanem LG, Abbott DH. Children born to women with polycystic ovary syndrome-short- and long-term impacts on health and development. Fertil Steril. 2019; 111:1065-75.

5. Dumesic DA, Goodarzi MO, Chazenbalk GD, Abbott DH. Intrauterine environment and polycystic ovary syndrome. Semin Reprod Med. 2014; 32: 159-65.

6. Mailath-Pokorny M, Kasprian G, Mitter C, Schopf V, Nemec U, Prayer D. Magnetic resonance methods in fetal neurology. Semin Fetal Neonatal Med. 2012; 17:278-84

7. Zhang Q, Bao Z-k, Deng M-x, Xu Q, Ding D-d, Pan M-m et al. Fetal growth fetal development, and placental features in women with polycystic ovary syndrome: analysis based on fetal and placental magnetic resonance imaging. Journal of Zhejiang University-SCIENCE B. 2020; 21:977-89.

8. Consensus REA-SP, Group W. Revised 2003 consensus on diagnostic criteria and long-term health risks related to polycystic ovary syndrome. Fertil Steril. 2004; 81:19-25.

9. Garel C. Fetal cerebral biometry: normal parenchymal findings and ventricular size. Eur Radiol. 2005; 15:809-13.

10. Tilea B, Alberti C, Adamsbaum C, Armoogum P, Oury JF, Cabrol D et al. Cerebral biometry in fetal magnetic resonance imaging: new reference data. Ultrasound Obstet Gynecol. 2009; 33:173-81.

11. Righini A, Zirpoli S, Parazzini C, Bianchini E, Scifo P, Sala C et al. Hippocampal infolding angle changes during brain development assessed by prenatal MR imaging. AJNR Am J Neuroradiol. 2006; 27:2093-7.

12. Sartor A, Arthurs O, Alberti C, Belarbi N, Tilea B, Boizeau P et al. Apparent diffusion coefficient measurements of the fetal brain during the third trimester of pregnancy: how reliable are they in clinical practice? Prenatal diagnosis. 2014; 34:357-66.

13. Nguyen The Tich S, Anderson PJ, Shimony JS, Hunt RW, Doyle LW, Inder TE. A novel quantitative simple brain metric using MR imaging for preterm infants. AJNR Am J Neuroradiol. 2009; 30:125-31.
14. Tich SN, Anderson PJ, Hunt RW, Lee KJ, Doyle LW, Inder TE. Neurodevelopmental and perinatal correlates of simple brain metrics in very preterm infants. Arch Pediatr Adolesc Med. 2011; 165:216-22.

15. Andescavage $\mathrm{N}$, duPlessis $\mathrm{A}$, Metzler M, Bulas D, Vezina $\mathrm{G}$, Jacobs $\mathrm{M}$ et al. In vivo assessment of placental and brain volumes in growth-restricted fetuses with and without fetal Doppler changes using quantitative 3D MRI. J Perinatol. 2017; 37:1278-84.

16. Kim MA, Han GH, Kim YH. Prediction of small-for-gestational age by fetal growth rate according to gestational age. PLoS One. 2019; 14:e0215737.

17. Palomba S, de Wilde MA, Falbo A, Koster MP, La Sala GB, Fauser BC. Pregnancy complications in women with polycystic ovary syndrome. Hum Reprod Update. 2015; 21:575-92.

18. Hjorth-Hansen A, Salvesen O, Engen Hanem LG, Eggebo T, Salvesen KA, Vanky E et al. Fetal Growth and Birth Anthropometrics in MetforminExposed Offspring Born to Mothers With PCOS. J Clin Endocrinol Metab. 2018; 103:740-47

19. Baillieux H, Smet HJD, Paquier PF, De Deyn PP, Mariën P. Cerebellar neurocognition: Insights into the bottom of the brain. Clinical Neurology and Neurosurgery. 2008; 110:763-73.

20. Xu F, Ge X, Shi Y, Zhang Z, Tang Y, Lin X et al. Morphometric development of the human fetal cerebellum during the early second trimester. Neuroimage. 2020; 207:116372.

21. Guimiot F, Garel C, Fallet-Bianco C, Menez F, Khung-Savatovsky S, Oury JF et al. Contribution of diffusion-weighted imaging in the evaluation of diffuse white matter ischemic lesions in fetuses: correlations with fetopathologic findings. AJNR Am J Neuroradiol. 2008; 29:110-5.

22. Yaniv G, Katorza E, Bercovitz R, Bergman D, Greenberg G, Biegon A et al. Region-specific changes in brain diffusivity in fetal isolated mild ventriculomegaly. Eur Radiol. 2016; 26:840-8.

23. Sanz-Cortes M, Egana-Ugrinovic G, Zupan R, Figueras F, Gratacos E. Brainstem and cerebellar differences and their association with neurobehavior in term small-for-gestational-age fetuses assessed by fetal MRI. Am J Obstet Gynecol. 2014; 210:452.e1-8.

24. Bell GA, Sundaram R, Mumford SL, Park H, Mills J, Bell EM et al. Maternal polycystic ovarian syndrome and early offspring development. Hum Reprod. 2018; 33:1307-15

25. Palomba S, Marotta R, Di Cello A, Russo T, Falbo A, Orio F et al. Pervasive developmental disorders in children of hyperandrogenic women with polycystic ovary syndrome: a longitudinal case-control study. Clin Endocrinol (Oxf). 2012; 77:898-904.

26. Cesta CE, Oberg AS, Ibrahimson A, Yusuf I, Larsson H, Almqvist C et al. Maternal polycystic ovary syndrome and risk of neuropsychiatric disorders in offspring: prenatal androgen exposure or genetic confounding? Psychol Med. 2020:50:616-24.

27. Norr ME, Hect JL, Lenniger CJ, Van den Heuvel M, Thomason ME. An examination of maternal prenatal BMI and human fetal brain development. J Child Psychol Psychiatry. 2021;62:458-69.

28. Peterson BS, Rosen T, Dingman S, Toth ZR, Sawardekar S, Hao X et al. Associations of Maternal Prenatal Drug Abuse With Measures of Newborn Brain Structure, Tissue Organization, and Metabolite Concentrations. JAMA Pediatr. 2020; 174:831-42.

29. Page KA, Luo S, Wang X, Chow T, Alves J, Buchanan TA et al. Children Exposed to Maternal Obesity or Gestational Diabetes Mellitus During Early Fetal Development Have Hypothalamic Alterations That Predict Future Weight Gain. Diabetes Care. 2019; 42:1473-80.

30. Kotini A, Koutlaki N, Anninos P, Liberis V, Hatzimihael A, Anastasiadis P. Fetal magnetoencephalography in intrauterine growth retarded pregnancies. Prenat Diagn. 2002; 22:1093-7.

\section{Publisher's Note}

Springer Nature remains neutral with regard to jurisdictional claims in published maps and institutional affiliations. 